\begin{tabular}{|c|l|}
\hline Title & MODULUS OF CONTINUITY OF THE DIRICHLET SOLUTIONS \\
\hline Author(s) & Aikawa, Hiroaki \\
\hline Citation & Hokkaido University Preprint Series in Mathematics, 946, 1-11 \\
\hline Issue Date & 2009-9-30 \\
\hline DOI & 10.14943/84093 \\
\hline Doc URL & http://hdl.handle.net/2115/69753 \\
\hline Type & bulletin (article) \\
\hline File Information & pre946.pdf \\
\hline
\end{tabular}

Instructions for use 


\title{
MODULUS OF CONTINUITY OF THE DIRICHLET SOLUTIONS
}

\author{
HIROAKI AIKAWA
}

\begin{abstract}
Let $D$ be a bounded domain in $\mathbb{R}^{n}$ with $n \geq 2$. For a function $f$ on $\partial D$ we denote by $\mathcal{H}^{D} f$ the Dirichlet solution of $f$ over $D$. It is classical that if $D$ is regular, then $\mathcal{H}^{D}$ maps the family of continuous boundary functions to the family of harmonic functions in $D$ continuous up to the boundary $\partial D$. We show that the better continuity of a boundary function $f$ ensures the better continuity of $\mathcal{H}^{D} f$ in the context of general modulus of continuity.
\end{abstract}

\section{INTRODUCTION}

Let $D$ be a bounded domain in $\mathbb{R}^{n}$ with $n \geq 2$. For a function $f$ on $\partial D$ we denote by $\mathcal{H}^{D} f$ the Dirichlet solution of $f$ over $D$, i.e., $\mathcal{H}^{D} f$ is harmonic in $D$ and $\mathcal{H}^{D} f=f$ on $\partial D$. It is well known that if $D$ is regular, then $\mathcal{H}^{D}$ maps the family of continuous boundary functions to the family of harmonic functions in $D$ continuous up to the boundary $\partial D$. It may be natural to think that the better continuity of a boundary function $f$ ensures the better continuity of $\mathcal{H}^{D} f$. Let $0<\beta \leq 1$. We say that $f$ is $\beta$-Hölder continuous if $|f(x)-f(y)| \leq C|x-y|^{\beta}$. Here, $C$ stands for an absolute positive constant whose value is unimportant and may change from one occurrence and the next. In the previous paper [1] we characterized the family of domains for which the $\beta$-Hölder continuity is preserved by $\mathcal{H}^{D}$. In particular, we showed that there is no domain which preserves the 1-Hölder (or Lipschitz) continuity. See [2] for further generalizations to $p$-harmonic functions in metric measure spaces.

This paper is devoted to investigation for similar problems in the context of general modulus of continuity. Let $\psi(t)$ be a positive nondecreasing function on $(0, \infty)$ with $\psi(0)=\lim _{t \rightarrow 0} \psi(t)=0$. In view of the Hölder continuity case, we assume that $\psi(t)$ is concave. See Section 5 for these assumptions. Let $\mathcal{M}$ be the family of positive nondecreasing concave functions $\psi(t)$ on $(0, \infty)$ with $\psi(0)=\lim _{t \rightarrow 0} \psi(t)=0$. Obviously, the Hölder

2000 Mathematics Subject Classification. 31A05, 31A20, 31B05, $31 \mathrm{~B} 25$.

Key words and phrases. Modulus of continuity, Dirichlet solution, concave function.

This work was supported in part by Kakenhi No.19654023 and No.20244007. 
continuity $\psi(t)=\min \left\{t^{\beta}, 1\right\}$ belongs to $\mathcal{M}$ for $0<\beta \leq 1$. For $\alpha>0$ let

$$
\psi_{\alpha}(t)= \begin{cases}(-\log t)^{-\alpha} & \text { for } 0<t<1 / e^{\alpha+1} \\ (\alpha+1)^{-\alpha} & \text { for } t \geq 1 / e^{\alpha+1}\end{cases}
$$

Then $\psi_{\alpha}(t) \in \mathcal{M}$. In fact, if $0<t<1 / e^{\alpha+1}$, then $\psi_{\alpha}^{\prime}(t)=\frac{\alpha}{t}(-\log t)^{-\alpha-1}>$ 0 and $\psi_{\alpha}^{\prime \prime}(t)=\frac{\alpha(-\log t)^{-\alpha-2}}{t^{2}}(\alpha+1+\log t)<0$. We see that $\psi_{\alpha}(t)$ is a modulus of continuity weaker than the Hölder continuity. We observe that $\mathcal{M}$ includes a lot of functions besides these two types of functions. See Proposition 2.1.

Let $\psi \in \mathcal{M}$. For an arbitrary set $E \subset \mathbb{R}^{n}, n \geq 2$, we consider the family $\Lambda_{\psi}(E)$ of all bounded continuous functions $f$ on $E$ with

$$
\|f\|_{\psi, E}=\sup _{x \in E}|f(x)|+\sup _{\substack{x, y \in E \\ x \neq y}} \frac{|f(x)-f(y)|}{\psi(|x-y|)}<\infty .
$$

Take another $\varphi \in \mathcal{M}$ and define the operator norm

$$
\left\|\mathcal{H}^{D}\right\|_{\psi \rightarrow \varphi}=\sup _{\substack{f \in \Lambda_{\psi}(\partial D) \\\|f\|_{\psi, \partial D} \neq 0}} \frac{\left\|\mathcal{H}^{D} f\right\|_{\varphi, D}}{\|f\|_{\psi, \partial D}} .
$$

The finiteness of $\left\|\mathcal{H}^{D}\right\|_{\psi \rightarrow \varphi}$ is of interest. Hinkkanen [3] considered the problem mainly for planar domains. However, the most interesting case $\psi=\varphi$ was treated only for the Hölder continuity. Our previous papers [1] and [2] also dealt with only the Hölder continuity.

The case $\psi=\varphi=\psi_{\alpha}$ with the above $\psi_{\alpha}$ appeared (at least tacitly) in connection with Kleinian groups. Shiga [6] and [5] proved a Hardy-Littlewood type theorem for holomorphic functions with respect to $\psi_{\alpha}(t)$. Let us state his result [5, Theorem 2.2], which is slightly changed for our disposal.

Theorem A. Let $f$ be a holomorphic function on the unit disk $\Delta$ and continuous on $\bar{\Delta}=\Delta \cup \partial \Delta$. Suppose that there exists $\alpha>0$ such that

$$
\left|f\left(e^{i \theta_{1}}\right)-f\left(e^{i \theta_{2}}\right)\right| \leq C \psi_{\alpha}\left(\left|\theta_{1}-\theta_{2}\right|\right) . \quad \text { for } 0 \leq \theta_{1}, \theta_{2}<2 \pi \text {. }
$$

Then

$$
\left|f^{\prime}(z)\right| \leq C \delta_{\Delta}(z)^{-1} \psi_{\alpha}\left(\delta_{\Delta}(z)\right) \quad \text { for } z, w \in \Delta,
$$

where $\delta_{\Delta}(z)=1-|z|=\operatorname{dist}(z, \partial \Delta)$.

Taking the real part, we obtain the harmonic counterpart: just simple replacement of "holomorphic" by "harmonic". Obviously, harmonic HardyLittlewood type result yields Theorem A immediately. Hardy-Littlewood type theorems and the modulus of continuity of the Dirichlet solution have 
close relationships. Proposition 2.3 below in Section 2 shows that Theorem A follows from

Theorem B. Let $D$ be the unit disk $\Delta$. Then $\left\|\mathcal{H}^{D}\right\|_{\psi \rightarrow \psi}<\infty$ for every $\psi(t)=\psi_{\alpha}(t)$ with $\alpha>0$.

Shiga [5] proved Theorem A by means of the explicit form of Cauchy's integral formula for the unit disk with the aid of very smart calculations. Our motivation is to establish a theorem which generalizes Theorem B with general domain and modulus of continuity. Since there is no explicit form of the Poisson integral for general domains, we need an approach different from Shiga's. One might think of Widman's estimates for the Green function [8]; these are not applicable because of the generality of domains and the subtleness of modulus of continuity. Instead, we shall apply barrier and harmonic measure method as in [1]. This method enables us to deal with not only $\psi_{\alpha}(t)$ but also general modulus of continuity $\psi \in \mathcal{M}$. It also reveals that the smoothness of the domain has no significance.

Without loss of generality, we may assume that $D$ is a bounded regular domain (see [1, Proposition 1]). For each $a \in \partial D$ we define a test function $\tau_{a, \psi}$ on $\partial D$ by

$$
\tau_{a, \psi}(\xi)=\psi(|\xi-a|) \quad \text { for } \xi \in \partial D .
$$

We shall see in Lemma 2.4 that $\tau_{a, \psi} \in \Lambda_{\psi}(\partial D)$.

Theorem 1.1. Let $\psi \in \mathcal{M}$. Then the following are equivalent:

(i) $\left\|\mathcal{H}^{D}\right\|_{\psi \rightarrow \psi}<\infty$.

(ii) There is a constant $C \geq 1$ such that

$$
\mathcal{H}^{D} \tau_{a, \psi}(x) \leq C \psi(|x-a|) \quad \text { for } x \in D,
$$

whenever $a \in \partial D$.

The second condition of Theorem 1.1 can be verified by using the harmonic measure. By $\omega(x, E, U)$ we denote the harmonic measure evaluated at $x$ of $E$ in $U$. We write $B(x, r)$ and $S(x, r)$ for the open ball and the sphere of center at $x$ and radius $r$, respectively.

Definition 1.2 (Global Harmonic Measure Decay property). We say that $D$ enjoys the Global Harmonic Measure Decay property with $\psi$ (abbreviated to the $\operatorname{GHMD}(\psi)$ property) if

$$
\omega(x, \partial D \backslash B(a, r), D) \leq C \frac{\psi(|x-a|)}{\psi(r)} \text { for } x \in D \cap B(a, r),
$$

whenever $a \in \partial D$ and $r>0$ is small. 
Theorem 1.3. Let $\psi \in \mathcal{M}$. If $\left\|\mathcal{H}^{D}\right\|_{\psi \rightarrow \psi}<\infty$, then D satisfies the $G H M D(\psi)$ property. Conversely, let $\Psi \in \mathcal{M}$ and suppose that there is $r_{0}>0$ such that

$$
\int_{r}^{r_{0}} \frac{d \psi(t)}{\Psi(t)} \leq C \frac{\psi(r)}{\Psi(r)} \text { for } 0<r<r_{0} .
$$

If D satisfies the $G H M D(\Psi)$ property, then $\left\|\mathcal{H}^{D}\right\|_{\psi \rightarrow \psi}<\infty$.

Corollary 1.4. Let $0<\alpha<\alpha^{\prime}$. If D satisfies the $\operatorname{GHMD}\left(\psi_{\alpha^{\prime}}\right)$ property, then $\left\|\mathcal{H}^{D}\right\|_{\psi \rightarrow \psi}<\infty$ with $\psi(t)=\psi_{\alpha}(t)$.

More geometrically we have the following corollaries.

Corollary 1.5. Let $D \subset \mathbb{R}^{n}, n \geq 2$, be a Lipschitz domain. Then $\left\|\mathcal{H}^{D}\right\|_{\psi \rightarrow \psi}<$ $\infty$ for every $\psi(t)=\psi_{\alpha}(t)$ with $\alpha>0$.

Corollary 1.6. Let $D \subset \mathbb{R}^{2}$ be a finitely connected plane domain with no puncture. Then $\left\|\mathcal{H}^{D}\right\|_{\psi \rightarrow \psi}<\infty$ for every $\psi(t)=\psi_{\alpha}(t)$ with $\alpha>0$.

Remark 1.7. The above corollary gives an extension of Theorem B and hence an alternative proof of Theorem A.

The plan of this paper is as follows. In the next section we shall prove Theorem 1.1 after preliminary observations for $\mathcal{M}$. In Section 3 we shall show Theorem 1.3 and Corollary 1.4. In Section 4 we shall give further notes for the harmonic measure decay property and $\mathcal{M}$, which derive Corollary 1.5 and Corollary 1.6. Finally, we shall give an observation, due to $\mathrm{S}$. T. Kuroda [4], for the relevance of the class $\mathcal{M}$, which may be of independent interest.

Acknowledgments. The author would like to thank Shiga for showing his preprints [6] and [5]. These give a starting point of this paper. The author also would like to thank Kuroda for showing his preprint [4].

\section{Proof of Theorem 1.1}

Let us begin with elementary observations.

Proposition 2.1. Let $\varphi, \psi \in \mathcal{M}$. Then $\varphi \circ \psi \in \mathcal{M}$.

Proof. It is easy to see that $\varphi \circ \psi$ is a nondecreasing function and that $\varphi \circ$ $\psi(0)=0$. For the concavity, let $s, t>0$ and $0<\lambda<1$. Then

$$
\begin{aligned}
\lambda \varphi \circ \psi(s)+(1-\lambda) \varphi \circ \psi(t) & \leq \varphi(\lambda \psi(s))+(1-\lambda) \varphi(\psi(t))) \\
& \leq \varphi(\lambda \psi(s)+(1-\lambda) \psi(t)) \leq \varphi(\psi(\lambda s+(1-\lambda) t)),
\end{aligned}
$$

where the concavity of $\varphi$, the monotonicity of $\varphi$ and the concavity of $\psi$ are used in this order. Thus the concavity of $\varphi \circ \psi$ follows, and hence $\varphi \circ \psi \in \mathcal{M}$. 
Lemma 2.2. Let $\psi \in \mathcal{M}$. Then

(i) $\psi(t) / t$ is nonincreasing, i.e., if $0<s<t$, then $\frac{\psi(s)}{s} \geq \frac{\psi(t)}{t}$.

(ii) $\psi$ is subadditive, i.e. if $s, t>0$, then $\psi(s+t) \leq \psi(s)+\psi(t)$.

(iii) $\psi(t)$ is doubling, i.e., If $0<s \leq t \leq 2 s$, then $\psi(s) \leq \psi(t) \leq 2 \psi(s)$.

Proof. (i) Since $\psi(0)=0$, the concavity of $\psi$ proves (i).

(ii) Let $f(t)=\psi(t) / t$. This is a nonincreasing function by (i). If $s, t>0$, then

$$
\begin{aligned}
\psi(s)+\psi(t)-\psi(s+t) & =s f(s)+t f(t)-(s+t) f(s+t) \\
& =s(f(s)-f(s+t))+t(f(t)-f(s+t)) \geq 0
\end{aligned}
$$

Hence (ii) follows.

(iii) Let $0<s \leq t \leq 2 s$. It follows from (i) that

$$
\frac{\psi(s)}{s} \geq \frac{\psi(t)}{t} \geq \frac{\psi(2 s)}{2 s}
$$

so that

$$
\psi(s) \leq \psi(t) \leq \psi(2 s) \leq 2 \psi(s),
$$

since $\psi(t)$ is nondecreasing. Thus (iii) follows.

Let $\delta_{D}(x)=\operatorname{dist}(x, \partial D)$.

Proposition 2.3. Let $\psi \in \mathcal{M}$. Suppose $h$ is a harmonic function on D. If

$$
|h(x)-h(y)| \leq C \psi(|x-y|) \quad \text { for } x, y \in D,
$$

then

$$
|\nabla h(x)| \leq C \delta_{D}(x)^{-1} \psi\left(\delta_{D}(x)\right) \quad \text { for } x \in D .
$$

Proof. Let $x \in D$. By assumption

$$
|h(x)-h(y)| \leq C \psi\left(\delta_{D}(x)\right) \quad \text { for } y \in B\left(x, \frac{1}{2} \delta_{D}(x)\right),
$$

where the doubling property of $\psi$ is used. Represent $h$ as the Poisson integral on $B\left(x, \frac{1}{2} \delta_{D}(x)\right)$ and differentiate it under the integral sign. Then we obtain

$$
|\nabla h(y)| \leq C \delta_{D}(x)^{-1} \psi\left(\delta_{D}(x)\right) \quad \text { for } y \in B\left(x, \frac{1}{4} \delta_{D}(x)\right) .
$$

Hence (2.1) holds.

Lemma 2.4. Let $\psi \in \mathcal{M}$ and let $\tau_{a, \psi}$ be as above. Then $\tau_{a, \psi} \in \Lambda_{\psi}(\partial D)$ and there is $C$ independent of $a \in \partial D$ such that

$$
\left\|\tau_{a, \psi}\right\|_{\psi, \partial D} \leq C
$$


Proof. By definition

$$
\sup _{\xi \in \partial D}\left|\tau_{a, \psi}(\xi)\right| \leq \psi(\operatorname{diam}(D))<\infty .
$$

Let $\xi, \xi^{\prime} \in \partial D$. Let us estimate $\left|\tau_{a, \psi}(\xi)-\tau_{a, \psi}\left(\xi^{\prime}\right)\right|=\left|\psi(|\xi-a|)-\psi\left(\left|\xi^{\prime}-a\right|\right)\right|$. Put $t=|\xi-a|$ and $s=\left|\xi^{\prime}-a\right|$. Without loss of generality we may assume that $0<s<t$. It follows from Lemma 2.2 (ii) and the monotonicity that $\left|\tau_{a, \psi}(\xi)-\tau_{a, \psi}\left(\xi^{\prime}\right)\right| \leq|\psi(t)-\psi(s)| \leq \psi(t-s)+\psi(s)-\psi(s)=\psi(t-s) \leq \psi\left(\left|\xi-\xi^{\prime}\right|\right)$, since $t-s \leq\left|\xi-\xi^{\prime}\right|$. Hence

$$
\frac{\left|\tau_{a, \psi}(\xi)-\tau_{a, \psi}\left(\xi^{\prime}\right)\right|}{\psi\left(\left|\xi-\xi^{\prime}\right|\right)} \leq 1
$$

Thus $\tau_{a, \psi} \in \Lambda_{\psi}(\partial D)$ and $\left\|\tau_{a, \psi}\right\|_{\psi, \partial D} \leq \psi(\operatorname{diam}(D))+1<\infty$.

Proof of Theorem 1.1. (i) $\Longrightarrow$ (ii). Suppose $\left\|\mathcal{H}^{D}\right\|_{\psi \rightarrow \psi}<\infty$. Then Lemma 2.4 gives

$$
\left\|\mathcal{H}^{D} \tau_{a, \psi}\right\|_{\psi, D} \leq\left\|\mathcal{H}^{D}\right\|_{\psi \rightarrow \psi}\left\|\tau_{a, \psi}\right\|_{\psi, \partial D} \leq C\left\|\mathcal{H}^{D}\right\|_{\psi \rightarrow \psi}<\infty .
$$

Hence

$$
\left|\mathcal{H}^{D} \tau_{a, \psi}(x)-\mathcal{H}^{D} \tau_{a, \psi}(y)\right| \leq C \psi(|x-y|) \quad \text { for } x, y \in D .
$$

Let $x^{*} \in \partial D$ be a point such that $\delta_{D}(x)=\left|x-x^{*}\right|$. Letting $y \rightarrow x^{*}$, we obtain $\mathcal{H}^{D} \tau_{a, \psi}(x) \leq C \psi\left(\delta_{D}(x)\right)$. Thus (ii) follows.

(ii) $\Longrightarrow$ (i). Suppose (ii) holds. Let $f \in \Lambda_{\psi}(\partial D)$ and let $x, y \in D$. It is sufficient to show that

$$
\left|\mathcal{H}^{D} f(x)-\mathcal{H}^{D} f(y)\right| \leq C\|f\|_{\psi, \partial D} \psi(|x-y|) .
$$

Without loss of generality, we may assume that $0<\delta_{D}(y) \leq \delta_{D}(x)$. Let $x^{*}, y^{*} \in \partial D$ be points such that $\delta_{D}(x)=\left|x-x^{*}\right|$ and $\delta_{D}(y)=\left|y-y^{*}\right|$. Let $f_{0}(\xi)=f(\xi)-f\left(x^{*}\right)$. It is easy to see that $\left\|f_{0}\right\|_{\psi, \partial D} \leq 2\|f\|_{\psi, \partial D}<\infty$ and

$$
\left|f_{0}(\xi)\right| \leq\|f\|_{\psi, \partial D} \psi\left(\left|\xi-x^{*}\right|\right)=\|f\|_{\psi, \partial D} \tau_{x^{*}, \psi}(\xi) .
$$

Let us consider two cases.

Case $1 .|x-y| \leq \frac{1}{2} \delta_{D}(x)$. Let us estimate $\nabla \mathcal{H}^{D} f=\nabla \mathcal{H}^{D} f_{0}$ on $B\left(x, \frac{2}{3} \delta_{D}(x)\right)$. If $z \in B\left(x, \frac{2}{3} \delta_{D}(x)\right)$, then (ii) and (2.2) give $\left|\mathcal{H}^{D} f_{0}(z)\right| \leq C\|f\|_{\psi, \partial D} \mathcal{H}^{D} \tau_{x^{*}, \psi}(z) \leq C\|f\|_{\psi, \partial D} \psi\left(\left|z-x^{*}\right|\right) \leq C\|f\|_{\psi, \partial D} \psi\left(\delta_{D}(x)\right)$, where the doubling property of $\psi$ is used in the last inequality. Representing $\mathcal{H}^{D} f_{0}$ as the Poisson integral and differentiating under the integral sign, we obtain

$$
\left|\nabla \mathcal{H}^{D} f(z)\right|=\left|\nabla \mathcal{H}^{D} f_{0}(z)\right| \leq C\|f\|_{\psi, \partial D} \frac{\psi\left(\delta_{D}(x)\right)}{\delta_{D}(x)} \quad \text { for } z \in B\left(x, \frac{1}{2} \delta_{D}(x)\right) .
$$


The mean value theorem gives

$$
\left|\mathcal{H}^{D} f(x)-\mathcal{H}^{D} f(y)\right| \leq C\|f\|_{\psi, \partial D} \frac{\psi\left(\delta_{D}(x)\right)}{\delta_{D}(x)}|x-y| \leq C\|f\|_{\psi, \partial D} \psi\left(\delta_{D}(x)\right) .
$$

Thus (i) follows in this case.

Case 2. $|x-y|>\frac{1}{2} \delta_{D}(x)$. By assumption we have $|x-y|>\frac{1}{2} \delta_{D}(x) \geq$ $\frac{1}{2} \delta_{D}(y)$. It is easy to see that

$$
\left|x^{*}-y^{*}\right| \leq|x-y|+\delta_{D}(x)+\delta_{D}(y) \leq 5|x-y| .
$$

Since $D$ is regular, it follows from (ii) and (2.2) that

$$
\begin{aligned}
& \left|\mathcal{H}^{D} f(x)-f\left(x^{*}\right)\right|=\left|\mathcal{H}^{D} f_{0}(x)\right| \\
& \leq C\|f\|_{\psi, \partial D} \mathcal{H}^{D} \tau_{x^{*}, \psi}(x) \leq C\|f\|_{\psi, \partial D} \psi\left(\delta_{D}(x)\right) \leq C\|f\|_{\psi, \partial D} \psi(|x-y|),
\end{aligned}
$$

where the doubling property of $\psi$ is used in the last inequality. Similarly, we have

$$
\left|\mathcal{H}^{D} f(y)-f\left(y^{*}\right)\right| \leq C|| f \|_{\psi, \partial D} \psi(|x-y|) .
$$

The doubling property of $\psi$ gives again

$$
\left|f\left(x^{*}\right)-f\left(y^{*}\right)\right| \leq\|f\|_{\psi, \partial D} \psi\left(\left|x^{*}-y^{*}\right|\right) \leq C\|f\|_{\psi, \partial D} \psi(|x-y|) .
$$

Collecting the above inequalities, we obtain

$$
\left|\mathcal{H}^{D} f(x)-\mathcal{H}^{D} f(y)\right| \leq C\|f\|_{\psi, \partial D} \psi(|x-y|) .
$$

Thus (i) follows.

\section{Proofs of Theorem 1.3 and Corollary 1.4}

Proof of Theorem 1.3. The first assertion of the theorem is easy. Let us prove the second assertion. Because of the local nature we may assume that $r_{0}=\infty$ in (1.3), i.e., we may assume that

$$
\int_{r}^{\infty} \frac{d \psi(t)}{\Psi(t)} \leq C \frac{\psi(r)}{\Psi(r)} \text { for } 0<r<\infty .
$$

Now let $a \in \partial D$ and $x \in \partial D$. Let $r=|x-a|$. Define an increasing sequence $\left\{r_{j}\right\}$ by $\psi\left(r_{j}\right)=2^{j} \psi(r)$. Then

$$
\begin{aligned}
\int_{r}^{\infty} \frac{d \psi(t)}{\Psi(t)} & =\sum_{j=0}^{\infty} \int_{r_{j}}^{r_{j+1}} \frac{d \psi(t)}{\Psi(t)} \geq \sum_{j=0}^{\infty} \frac{1}{\Psi\left(r_{j+1}\right)} \int_{r_{j}}^{r_{j+1}} d \psi(t) \\
& =\sum_{j=0}^{\infty} \frac{\psi\left(r_{j+1}\right)-\psi\left(r_{j}\right)}{\Psi\left(r_{j+1}\right)}=\sum_{j=0}^{\infty} \frac{2^{j} \psi\left(r_{j}\right)}{\Psi\left(r_{j+1}\right)}=\frac{1}{4} \sum_{j=1}^{\infty} \frac{\psi\left(r_{j+1}\right)}{\Psi\left(r_{j}\right)},
\end{aligned}
$$


where the definition of $r_{j}$ is used in the last two equalities. By definition

$$
\begin{aligned}
\mathcal{H}^{D} \tau_{a, \psi}(x) & \leq \mathcal{H}^{D}\left(\psi(r) \chi_{\partial D \cap B(a, r)}\right)(x)+\sum_{j=0}^{\infty} \psi\left(r_{j+1}\right) \mathcal{H}^{D}\left(\chi_{\partial D \backslash B\left(a, r_{j}\right)}\right)(x) \\
& \leq \psi(r)+\sum_{j=0}^{\infty} \psi\left(r_{j+1}\right) \omega\left(x, \partial D \backslash B\left(a, r_{j}\right), D\right) \\
& \leq \psi(r)+\sum_{j=0}^{\infty} \psi\left(r_{j+1}\right) \frac{\Psi(r)}{\Psi\left(r_{j}\right)} \\
& =\psi(r)+\psi\left(r_{1}\right) \frac{\Psi(r)}{\Psi\left(r_{0}\right)}+\sum_{j=1}^{\infty} \psi\left(r_{j+1}\right) \frac{\Psi(r)}{\Psi\left(r_{j}\right)} \\
& \leq 3 \psi(r)+4 \Psi(r) \int_{r}^{\infty} \frac{d \psi(t)}{\Psi(t)}
\end{aligned}
$$

where the last inequality follows from $r_{0}=r, \psi\left(r_{1}\right)=2 \psi(r)$ and (3.2). By (3.1) we have

$$
\mathcal{H}^{D} \tau_{a, \psi}(x) \leq 3 \psi(r)+C \Psi(r) \frac{\psi(r)}{\Psi(r)} \leq C \psi(r) .
$$

Thus (ii) in Theorem 1.1 holds, and hence $\left\|\mathcal{H}^{D}\right\|_{\psi \rightarrow \psi}<\infty$.

Proof of Corollary 1.4. Let $0<\alpha<\alpha^{\prime}$. Let $0<r<1 / e^{\alpha+1}$. Then

$$
\int_{r}^{1 / e^{\alpha+1}} \frac{d \psi_{\alpha}(t)}{\psi_{\alpha^{\prime}}(t)}=\alpha \int_{r}^{1 / e^{\alpha+1}} \frac{(\log 1 / t)^{-\alpha-1}}{(\log 1 / t)^{-\alpha^{\prime}}} \frac{d t}{t}=\alpha \int_{r}^{1 / e^{\alpha+1}}\left(\log \frac{1}{t}\right)^{\alpha^{\prime}-\alpha-1} \frac{d t}{t} .
$$

Letting $s=\log 1 / t$, we obtain that this integral is equal to

$\alpha \int_{\alpha+1}^{\log 1 / r} s^{\alpha^{\prime}-\alpha-1} d s=\frac{\alpha}{\alpha^{\prime}-\alpha}\left[s^{\alpha^{\prime}-\alpha}\right]_{\alpha+1}^{\log 1 / r} \leq \frac{\alpha}{\alpha^{\prime}-\alpha}\left(\log \frac{1}{r}\right)^{\alpha^{\prime}-\alpha}=\frac{\alpha}{\alpha^{\prime}-\alpha} \frac{\psi_{\alpha}(r)}{\psi_{\alpha^{\prime}}(r)}$.

Thus (1.3) holds. Hence Theorem 1.3 proves $\left\|\mathcal{H}^{D}\right\|_{\psi \rightarrow \psi}<\infty$.

\section{Proofs of Corollary 1.5 and Corollary 1.6}

Let us begin with observations of the harmonic measure decay property.

Definition 4.1 (Local Harmonic Measure Decay property). We say that $D$ enjoys the Local Harmonic Measure Decay property with $\psi$ (abbreviated to the $\operatorname{LHMD}(\psi)$ property) if

$$
\omega(x, D \cap S(a, r), D \cap B(a, r)) \leq C \frac{\psi(|x-a|)}{\psi(r)} \quad \text { for } x \in D \cap B(a, r),
$$

whenever $a \in \partial D$ and $r>0$ is small. 
Remark 4.2. Let $\psi \in \mathcal{M}$. If the $\operatorname{LHMD}(\psi)$ property holds for $D$, then so does the $\operatorname{GHMD}(\psi)$ property. If $\psi(t)=t^{\alpha}$, then the converse is true as well (see [1]). For general $\psi \in \mathcal{M}$, we do not know whether the converse holds or not.

Remark 4.3. See Sugawa [7] for the plane case.

Remark 4.4. If $\psi(t)=t^{\beta}$, then we can replace $\psi(|x-a|) / \psi(r)$ by $\psi(|x-a| / r)$ in (1.2) and (4.1). For general $\psi \in \mathcal{M}$, however, such a replacement is not valid because of the inhomogeneity of $\psi$.

It is convenient to introduce a certain order among functions in $\mathcal{M}$.

Definition 4.5. Let $\varphi, \psi \in \mathcal{M}$. We say that $\varphi \lesssim \psi$ if there are $r_{0}>0$ and $C>0$ such that

$$
\frac{\varphi(s)}{\varphi(r)} \leq C \frac{\psi(s)}{\psi(r)} \quad \text { for } 0<s<r \leq r_{0} .
$$

The definition of the GHMD property readily gives

Proposition 4.6. Let $\varphi, \psi \in \mathcal{M}$ and let $\varphi \lesssim \psi$. If the $G H M D(\varphi)$ property holds for $D$, then so does the GHMD $(\psi)$ property.

The order $\lesssim$ and the composition have the following relationship.

Proposition 4.7. Let $\varphi, \psi \in \mathcal{M}$. Then $\psi \lesssim \varphi \circ \psi$.

Proof. Let $0<s<r$. Put $S=\psi(s)$ and $R=\psi(r)$. Since $0<s \leq R$ from the monotonicity of $\psi$, it follows from Lemma 2.2 (i) that

$$
\frac{\varphi(\psi(r))}{\psi(r)}=\frac{\varphi(R)}{R} \leq \frac{\varphi(S)}{S}=\frac{\varphi(\psi(s))}{\psi(s)} .
$$

Hence

$$
\frac{\psi(s)}{\psi(r)} \leq \frac{\varphi(\psi(s))}{\varphi(\psi(r))}=\frac{\varphi \circ \psi(s)}{\varphi \circ \psi(r)}
$$

Let $\alpha>0$ and $\beta>0$. Then $\psi_{\alpha}\left(t^{\beta}\right)=\beta^{-\alpha} \psi_{\alpha}(t)$, so that $t^{\beta} \lesssim \psi_{\alpha}(t)$ by Proposition 4.7. Hence Corollary 1.4 and Proposition 4.6 give

Corollary 4.8. Let $\beta>0$. If $D$ satisfies the $G H M D\left(t^{\beta}\right)$ property, then $\left\|\mathcal{H}^{D}\right\|_{\psi \rightarrow \psi}<\infty$ for every $\psi(t)=\psi_{\alpha}(t)$ with $\alpha>0$.

Proof of Corollary 1.5. It is well-known that every Lipschitz domain $D$ satisfies the $\operatorname{LHMD}\left(t^{\beta}\right)$ property for some $\beta>0$, and so the $\operatorname{GHMD}\left(t^{\beta}\right)$ property. Hence Corollary 4.8 gives Corollary 1.5.

Proof of Corollary 1.6. We observe that a finitely connected plane domain with no puncture satisfies the $\operatorname{GHMD}\left(t^{\beta}\right)$ property for $0<\beta<1 / 2$. Hence Corollary 4.8 gives Corollary 1.6. 


\section{REMARK}

In Section 1 we have assumed that $\psi(t)$ is concave. We observe that this assumption can be essentially relaxed to the nonincreasing property of $\psi(t) / t$. This observation is due to S. T. Kuroda [4]. We include it with his permission.

Proposition 5.1. Let $\psi(t)$ be a positive nondecreasing function on $(0, \infty)$ with $\psi(0)=\lim _{t \rightarrow 0} \psi(t)=0$. Suppose that $\psi(t) / t$ is nonincreasing. By $\mathcal{U}_{\psi}$ we denote the family of concave functions $\varphi$ on $(0, \infty)$ such that $\psi \leq \varphi$. Let

$$
\Psi(t)=\inf \left\{\varphi(t): \varphi \in \mathcal{U}_{\psi}\right\} \quad \text { for } t>0
$$

be the concave upper envelope of $\psi$. Then

(i) $\Psi$ is a concave nondecreasing function on on $(0, \infty)$ with $\Psi(0)=0$.

(ii) $\frac{1}{2} \Psi(t) \leq \psi(t) \leq \Psi(t)$ for $t>0$.

Proof. (i) We prove only the concavity of $\Psi$ as the remaining is easy. Let $s>0$ and $t>0$ and let $0<\lambda<1$. Then for every $\varphi \in \mathcal{U}_{\psi}$ we have

$$
\lambda \varphi(s)+(1-\lambda) \varphi(t) \leq \varphi(\lambda s+(1-\lambda) t)) .
$$

Taking the infimum for $\varphi \in \mathcal{U}_{\psi}$, we obtain

$$
\begin{aligned}
\lambda \Psi(s)+(1-\lambda) \Psi(t) & =\lambda \inf _{\varphi \in \mathcal{U}_{\psi}} \varphi(s)+(1-\lambda) \inf _{\varphi \in \mathcal{U}_{\psi}} \varphi(t) \\
& \leq \inf _{\varphi \in \mathcal{U}_{\psi}}(\lambda \varphi(s)+(1-\lambda) \varphi(t)) \\
& \leq \inf _{\varphi \in \mathcal{U}_{\psi}} \varphi(\lambda s+(1-\lambda) t)=\Psi(\lambda s+(1-\lambda) t) .
\end{aligned}
$$

Thus $\Psi$ is a concave function.

(ii) Let us prove the first inequality since the second is obvious. Let $t_{0}>$ 0 . If $\psi\left(t_{0}\right)=\Psi\left(t_{0}\right)$, then there is nothing to prove. Suppose $\psi\left(t_{0}\right)<\Psi\left(t_{0}\right)$. By definition we infer that $\Psi(t)$ is a linear function in a neighborhood of $t_{0}$. Let $\left(t_{1}, t_{2}\right)$ be the largest interval including $t_{0}$ and on which $\psi(t)<\Psi(t)$. Then $\Psi(t)$ is linear on $\left[t_{1}, t_{2}\right], \Psi\left(t_{1}\right)=\psi\left(t_{1}\right)$ and $\Psi\left(t_{2}\right)=\psi\left(t_{2}\right)$. Let $p(t)$ be the linear function whose graph is the line connecting the points $\left(t_{1}, \psi\left(t_{1}\right)\right)$ and $\left(t_{2}, \psi\left(t_{2}\right)\right)$. Let $q(t)$ be the linear function whose graph is the line connecting the points $\left(0, \psi\left(t_{0}\right)\right)$ and $\left(t_{0}, \Psi\left(t_{0}\right)\right)$. We observe that $p\left(t_{0}\right)=q\left(t_{0}\right)=\Psi\left(t_{0}\right)$ and $p(0) \leq \psi\left(t_{0}\right)=q(0)$, and hence

$$
q(t) \leq p(t) \quad \text { for } t \geq t_{0} .
$$

See Figure 1. Evaluate $p(t)$ and $q(t)$ at $t=t_{2}$. Then the explicit form $q(t)=t_{0}^{-1}\left(p\left(t_{0}\right)-\psi\left(t_{0}\right)\right)\left(t-t_{0}\right)+p\left(t_{0}\right)$ gives

$$
\frac{p\left(t_{0}\right)-\psi\left(t_{0}\right)}{t_{0}}\left(t_{2}-t_{0}\right)+p\left(t_{0}\right) \leq p\left(t_{2}\right)=\psi\left(t_{2}\right),
$$




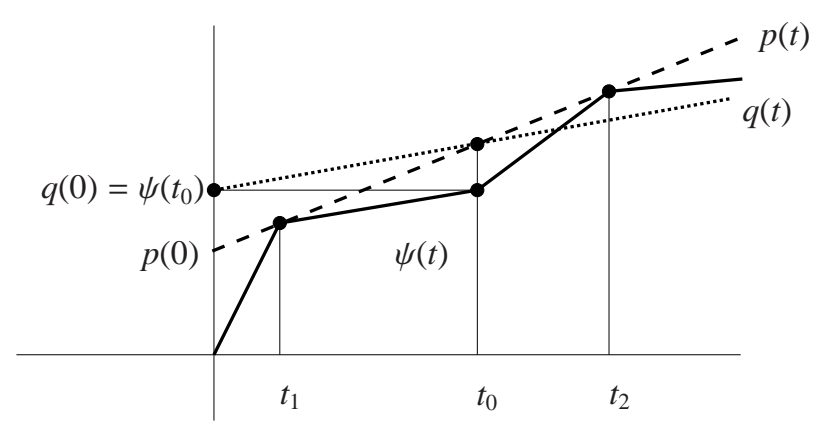

FIGURE 1. The concave upper envelope.

so that

$$
\frac{t_{2}}{t_{0}} p\left(t_{0}\right)=\left(\frac{t_{2}-t_{0}}{t_{0}}+1\right) p\left(t_{0}\right) \leq \frac{t_{2}-t_{0}}{t_{0}} \psi\left(t_{0}\right)+\psi\left(t_{2}\right) .
$$

Since $\psi(t) / t$ is nonincreasing, it follows that

$$
\Psi\left(t_{0}\right)=p\left(t_{0}\right) \leq \frac{t_{2}-t_{0}}{t_{2}} \psi\left(t_{0}\right)+\frac{t_{0}}{t_{2}} \psi\left(t_{2}\right) \leq \psi\left(t_{0}\right)+t_{0} \frac{\psi\left(t_{0}\right)}{t_{0}}=2 \psi\left(t_{0}\right) .
$$

Thus the first inequality of (ii) follows.

Remark 5.2. The nonincreasing property of $\psi(t) / t$ in the assumptions of the proposition can be replaced by the subadditivity of $\psi$. See Lemma 2.2 (ii).

\section{REFERENCES}

[1] H. Aikawa, Hölder continuity of the Dirichlet solution for a general domain, Bull. London Math. Soc. 34 (2002), no. 6, 691-702.

[2] H. Aikawa and N. Shanmugalingam, Hölder estimates of p-harmonic extension operators, J. Differential Equations 220 (2006), no. 1, 18-45.

[3] A. Hinkkanen, Modulus of continuity of harmonic functions, J. Analyse Math. 51 (1988), 1-29.

[4] S. T. Kuroda, Diagonalization modulo norm ideals; Voigt's method and modulus of continuity, preprint (2009).

[5] H. Shiga, Modulus of continuity, a Hardy-Littlewood theorem and its application, Infinite dimensional Teichmüller space and moduli space, RIMS Kôkyûroku Bessatsu, Res. Inst. Math. Sci. (RIMS), Kyoto, 2009.

[6] _ Riemann mappings of invariant components of Kleinian groups, J. London Math. Soc. (2) (2009).

[7] T. Sugawa, Uniformly perfect sets: analytic and geometric aspects, Sugaku Expositions 16 (2003), no. 2, 225-242.

[8] K.-O. Widman, Inequalities for the Green function and boundary continuity of the gradient of solutions of elliptic differential equations, Math. Scand. 21 (1967), 17-37 (1968).

Department of Mathematics, Hokkaido University, Sapporo 060-0810, JaPan

E-mail address: aik@math.sci.hokudai .ac.jp 